\title{
Optical and Mechanical Design of C-Mod Motional Stark Effect Diagnostic ${ }^{*}$
}

\author{
D. I. Simon ${ }^{1}$, N. L. Bretz ${ }^{1}$, E. Marmar ${ }^{2}$, R. Bravenec ${ }^{3}$, and R. F. Parsells ${ }^{1}$.
}

\begin{abstract}
A Motional Stark Effect (MSE) instrument is being installed on the Alcator C-Mod tokamak at MIT. This MSE diagnostic will provide measurements of the spatial profile of the internal poloidal magnetic field. The MSE has its primary collection optics inside the vacuum vessel. The light collected by the internal optics passes through a vacuum window and is relayed to a fiber optic array. The MSE optics are shared by a Beam Emission Spectroscopy (BES) diagnostic which measures electron density fluctuations and their spatial correlations. This optical system requires high throughput and spatial resolution of less than $1 \mathrm{~cm}$ at the focal plane in the plasma. The design requirements for the internal optics also include the effects associated with plasma impingement, plasma disruptions, and thermal excursions. The parameters that affect polarization measurement include the location and orientation of optical elements, the choice of substrates and optical materials. These unique design requirements led to a number of interesting optical and mechanical design features which are presented here.
\end{abstract}

Index Terms-MSE, DNB, C-Mod.

\section{INTRODUCTION}

A Motional Stark Effect (MSE) instrument to measure the poloidal magnetic field profile using a neutral beam on CMod has been designed and partly installed. Optics view the perpendicular beam in the region $-0.3<\left(\mathrm{R}-\mathrm{R}_{\mathrm{o}}\right) / \mathrm{a}<$ 1.05. When the beam is apertured to a width of $2 \mathrm{~cm}$, the resulting radial resolution is $\sim 1 \mathrm{~cm}$ near the outer edge and $\sim 2 \mathrm{~cm}$ near the core. Statistical errors in the measurement of the poloidal to toroidal magnetic field ratio, $\mathrm{Bp} / \mathrm{Bt}$, are estimated to be less than $0.2 \%$ for $n_{\mathrm{eo}}<2.0 \times 10^{14} \mathrm{~cm}^{-3}$, similar to other MSE systems[1]. The primary collection optics reside within the vacuum chamber reflecting and imaging the neutral beam through a vacuum window so that $\mathrm{H}_{\alpha}$ emission can be used for both Beam Emission Spectroscopy (BES) and MSE. Low Verdet glass and dielectric mirrors are used to minimize polarization changes. Polarization will be measured by conventional

\footnotetext{
${ }^{1}$ Princeton University Plasma Physics Laboratory, P.O. Box 451, Princeton, NJ 08543.

${ }^{2}$ MIT Plasma Science and Fusion Center, 175 Albany Street, Cambridge, MA 02139.

${ }^{3}$ Fusion Research Center, University of Texas at Austin, RLM 11.222, Austin, TX 78712.
}

techniques[2] using photoelastic modulators, optical filters, and photomultipliers. Measurements at higher density are compromised by increased beam attenuation and Bremsstrahlung.

\section{OPTICS FOR MSE ON C-MOD}

The MSE optical system consists of sixteen optical elements, not counting the quartz vacuum window located in the middle of the system. Five lenses and three mirrors are located in the vacuum vessel while another five lenses, two photoelastic modulators (PEMs) and a fiber optic collection array are located outside the vessel. Lens material was chosen as Schott SFL6 for its small Verdet constant and the lenses are oriented to minimize polarization changes. The first lens is small (center thickness is $5 \mathrm{~mm}$ ), helping to minimize the Verdet effect from the toroidal field. The second set of lenses see a contribution due only to the vertical field, while the third set of lenses experience a smaller radial magnetic field contribution. The lenses are all anti-reflection coated with a multi-layer coating which minimizes polarization changes. The three dielectric mirrors are optimized to minimize polarization shifts at $656 \mathrm{~nm}$, the design wavelength. The aim is to limit optically induced changes in the polarization to about 0.1 degree, which is less than the required resolution of the MSE instrument.

\section{OptICS AND HOUSING INSIDE C-MOD}

All materials inside the C-Mod vacuum vessel were approved by the C-Mod vacuum group. The housing was constructed entirely of non-magnetic materials. The housing for the lens and mirror elements in the vacuum vessel was made with welded sections of stainless steel tubing (see Figure 1). Thin wall tubing ( $0.063^{\prime \prime}$ thick) was used to reduce eddy current forces.

The lenses were mounted in barrels using three point supports located $120^{\circ}$ apart. The supports are small Spectralon buttons designed to reduce mounting stress on the lenses. Custom stainless steel mirror mounts were made for each of the three mirrors. The mirrors rest on small Spectralon pads and are retained by low profile stainless steel clips.

The geometry of the housing was complicated by the need to view the neutral beam along a magnetic field line while 
maintaining a component parallel to the beam to Doppler shift the Stark components away from unshifted $\mathrm{H}_{\alpha}$ coming from the plasma edge. The MSE optics relay collected light out of the vessel through a $10 \mathrm{~cm}$ diameter quartz window on the same port but 9" above the neutral beam that it is imaging. The final factor determining the geometry is the need to avoid impinging on the plasma. Any stainless steel part in the optical path was darkened using a vacuum compatible hydrogen furnace heat treatment to suppress stray light. The MSE vacuum housing is bolted to the inside flange of the quartz viewport. A split ring bracket (see Figure 1) is employed to support the MSE and protect the diagnostic from a plasma disruption. A heat shield is used to protect the thin first lens. See Figures 1 and 2.

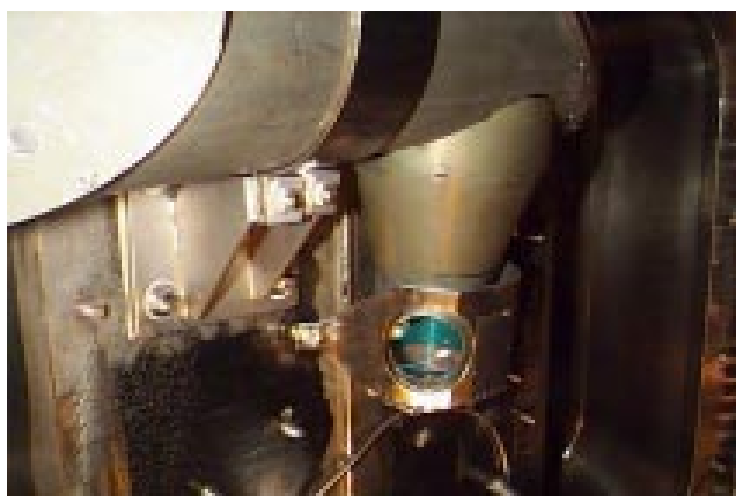

Figure 1. View of MSE from inside C-Mod showing input lens, vertical housing for second lens set, split ring support, and thin wall housing containing last two mirrors.

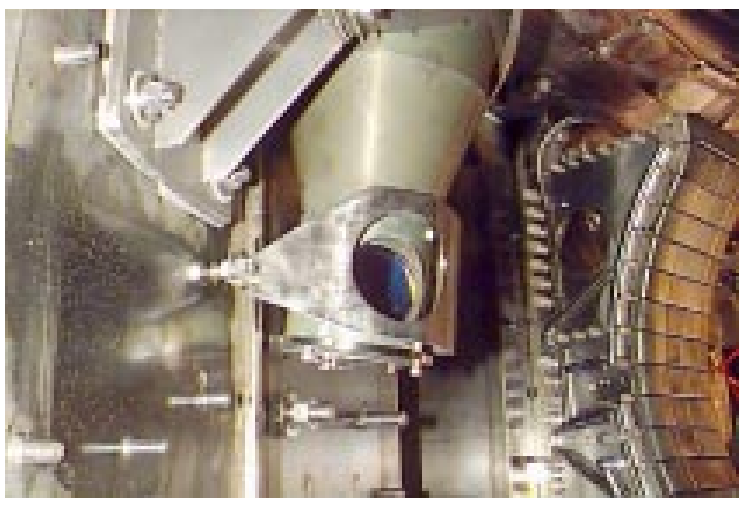

Figure 2. Close-up view from inside C-Mod of MSE showing input lens, heat shield and lower portion of split ring support bracket.

A stainless steel shutter (not shown) has been made but not yet installed. The shutter fits between the heat shield and the housing for the first lens. It is designed with an aperture for unobstructed viewing of the neutral beam and on the opposite side an aperture with a Polorcor polarizer. The shutter is designed to rotate $180^{\circ}$. A small braided monel wire wraps around the shutter and connects to a pair of linear feedthroughs on the outside of the vessel which drive the motion. The shutter rides on a small inconel ring located just below the heat shield (see Figure 2).

\section{OPTICS AND HOUSING OUTSIDE C-MOD}

The exterior optics are not yet installed on C-Mod. The exterior optics relay the light from an intermediate image plane just outside the quartz viewport to a more accessible location for the fiber optic array about $60 \mathrm{~cm}$ away. There is a set of crossed photoelectric modulators (PEMs) used in the polarization measurement located just before the last lens (see Figure 3). This final lens focuses the light to a concave image plane with a $19.5 \mathrm{~mm}$ radius of curvature. The image plane is populated with closely spaced bundles of $1 \mathrm{~mm}$ diameter fiber optics. The MSE fibers are bundled in sets of seven fibers and placed in stainless steel ferules with a $3.66 \mathrm{~mm}$ inside diameter. The BES fibers are bundled in sets of four fibers and placed in stainless steel ferules with a $3 \mathrm{~mm}$ inside diameter. A delrin and aluminum composite fixture containing a two dimensional set of holes locate the fibers in the $x-y$ plane. Delrin is used to provide friction to hold the ferrules in place, while the aluminum is needed to provide structural rigidity to the holes spaced as close as $0.25 \mathrm{~mm}$ apart. The location of the fibers along the z-axis is set by pushing the ferrules through the delrin and aluminum composite fixture until they contact a temporary convex fixture made from carefully positioned pins which matches the image plane.

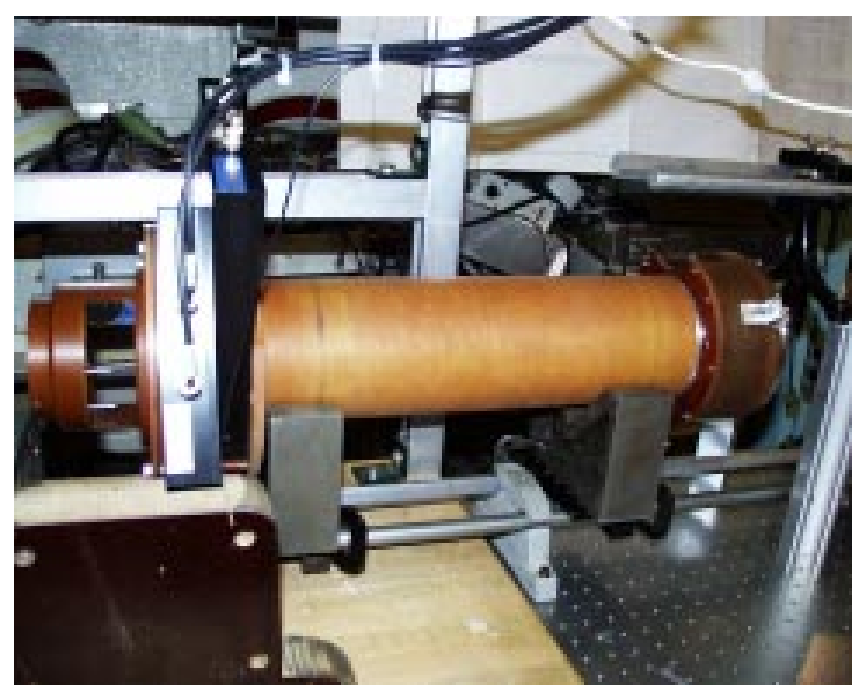

Figure 3. View of MSE external optics enclosure showing (from right) the phenolic window adapter and lens barrel, PEMs, and fiber optic array holder with cutout for temporary alignment fixture.

Outside the vacuum boundary there are high magnetic fields and restricted access. The exterior optics enclosure is made of phenolic elements in a lens barrel design. Spacers made of G-10 fix the location of the lenses inside the phenolic tube along the z-axiz. The lenses were mounted in G-10 barrels using a three point contact similar to that used for the interior lenses described above. The inside surface 
of the G-10 lens barrels are painted flat black to suppress stray light. A phenolic adapter was made to fit over the quartz vacuum window and bolts to the vacuum flange (see Figure 3). Circular phenolic plates, with cutouts to accommodate existing equipment on C-Mod, bracket the PEMs and maintain a $45^{\circ}$ displacement between their optical axis. The PEMs are located very close to the final lens in order to clear a cryogenic cowling surrounding CMod. An additional phenolic piece was made to hold the final lens, the fiber optic array fixture, and accommodate the temporary alignment fixture. The exterior optics are supported by a pair of aluminum braces, (not shown) placed just behind the PEMs, which in turn rest on the exterior neutral beam duct.

\section{SUMMARY}

The optics for a Motional Stark Effect (MSE) diagnostic have been partially installed on C-Mod. A number of environmental factors, like exposure to high magnetic fields and vacuum compatibility, along with inevitable space constraints inside and outside of the C-Mod vacuum vessel, strongly influenced the design of the MSE optics and housing. Material selection criteria for the optics and the housing were discussed. Methods for mounting and supporting the optics were also related. The exterior optics and housing are being tested at PPPL using a light source to simulate the neutral beam. When the testing is completed, we plan to finish the installation of MSE on C-Mod.

\section{ACKNOWLEDGMENTS}

${ }^{*}$ Work supported by U.S. Department of Energy under Contracts Nos. PPPL DE-AC02-76-3073 and MIT DEFC02-99ER54512

\section{REFERENCES}

[1] F. M. Levinton, G. M. Gammel, R. Kaita, H. W. Kugel, D. W. Robert, Rev. Sci. Instrum. 61, 2914 (1990).

[2] J. C. Kemp, J. Opt. Soc. Am. 59, 950 (1969). 